\title{
Algoritmo de seguimiento y planeación de trayectorias para un robot fertilizador en invernaderos
}

\section{Tracking algorithm and trajectory planning for a greenhouse fertilizer robot}

\author{
BETANZOS-CASTILLO, Francisco $\dagger^{*}$, BECERRA-AMBRIZ, María Cecilia, MORÁN- \\ HERNÁNDEZ, Juan y JIMÉNEZ-CAMPUZANO, Everardo
}

Tecnológico de Estudios Superiores de Valle de Bravo, División de Ingeniería en Mecatrónica, Valle de Bravo, México

ID $1^{\mathrm{er}}$ Autor: Francisco, Betanzos-Castillo / ORC ID: 0000-0002-7245-703X, CVU CONACYT ID: 206209

ID $1^{\text {er }}$ Coautor: María Cecilia, Becerra-Ambriz / ORC ID: 0000-0002-0322-7230, CVU CONACYT ID: 706715

ID $2^{\text {do }}$ Coautor: Juan, Morán-Hernández / ORC ID: 0000-0002-2677-1888, CVU CONACYT ID: 326278

ID $3^{\text {er }}$ Coautor: Everardo, Jiménez-Campuzano / ORC ID: 0000-0002-0392-3754, CVU CONACYT ID: 993207

DOI: $10.35429 / J C S .2019 .9 .3 .22 .26$

Recibido: 12 de Junio, 2019; Aceptado 30 de Septiembre, 2019

\begin{abstract}
Resumen
El uso de algoritmos es una actividad necesaria en el diseño y construcción de cualquier robot móvil. En este trabajo se propondrá un algoritmo escrito en Unity diseñado con la finalidad de simular el seguimiento de la trayectoria que trazará el robot móvil. Una de las aplicaciones que motivó este proyecto fue la aplicación en el sector agrícola para la distribución de fertilizantes. La robótica aplicada a la agricultura, aunque en México está en etapas tempranas de su desarrollo, de forma progresiva está interviniendo los invernaderos productivos. Por lo que es necesario proponer nuevas estrategias de automatización en el sector. Como parte de una etapa de diseño y modelado de un robot virtual, este proyecto propone un algoritmo de un prototipo de robot virtual 3D que permita visualizar y posicionarse en el plano $2 \mathrm{D}$ y así tener simulaciones preliminares para la posterior construcción del prototipo de Robot.
\end{abstract}

Robots, fertilización, Mecanización de la agricultura

\begin{abstract}
The use of algorithms is a necessary activity for the design and construction, of any mobile robot. This study proposes an algorithm for future farm applications. Fertilization is an aspect that represents a third of the production costs. Robotics applied to agriculture, although in Mexico it is in the early stages of its development, the productive greenhouses are progressively automatized. Therefore, it is necessary to propose new automation strategies in the sector. As part of a stage of design and modeling of a virtual robot, this project proposes an algorithm of a $3 \mathrm{D}$ virtual robot prototype that allows visualizing and positioning in the $2 \mathrm{D}$ plane and thus having preliminary simulations for the subsequent construction of the Robot prototype.
\end{abstract}

Robots, fertilization, Mechanization of agriculture

\footnotetext{
* Correspondencia al Autor: (fbetanzoscastillo@gmail.com)

$\dagger$ Investigador contribuyendo como primer autor.
} 


\section{Introducción}

En los inicios de la agricultura los campesinos utilizaban el poder de los animales para ahorrarse los trabajos forzosos que implica el cultivo y cuidado de los sistemas agrícolas productivos (Bonifaz, Happy, Linhares, \& Velasco, 2009). Para ejemplificar, se necesitan de 200 a 300 horas de trabajo o de mano de obra para producir 2 hectáreas con un arado para caminar, una rastra de matorrales, y una transmisión manual de semillas (Bellis, 2009).

Lo anterior y diversos trabajos sobre automatización de sistemas de invernadero como por ejemplo el realizado por Faizzan y colaboradores en donde se realiza un modelo a escala de invernadero pensado para el cultivo de vegetales de forma intradomiciliaria (Muhammad Faizan Siddiqui, Asim ur Rehman Khan, Neel Kanwal, Haider Mehdi, Aqib Noor, 2017), ponen de manifiesto la importancia de contribuir a la automatización de los sistemas agrícolas productivos para minimizar esfuerzos y aumentar la productividad.

La robótica agrícola está, al día de hoy, en fase de prototipo avanzado, en lo que se viene a denominar nivel 7 de madurez tecnológica, lo que equivale a capacidad de demostración de sistema o prototipo en un entorno real, por lo que, salvo algunas excepciones, aún no alcanza la madurez necesaria como para abordar su implantación real y extendida en el sector. Además de motivos económicos, que enfrentan el alto coste de parte de la tecnología utilizada con los costes de la mano de obra dedicada a las actividades agrícola, las dificultades tecnológicas derivadas principalmente de la falta de estructuración del entorno de trabajo, suponen un complejo reto a superar.

Una segunda problemática que enfrentan los países en desarrollo como México es que el desarrollo físico de sistemas automatizados, demandan inversión económica, para ello el modelado virtual previo a la construcción sirve para evitar gastos innecesarios y simular las características dinamicas en el diseño. Es por esto que este proyecto propone un algoritmo de seguimiento de trayectorias para un robot virtual con perspectivas de aplicación en invernaderos. Con esto se busca explorar modelos de diseño virtual de un robot fertilizador que permitan tener un punto de partida para la automatización de invernaderos.
A través del software Unity se diseñó un entorno virtual de invernadero que permitiese posicionar a un modelo de robot virtual dentro del mismo entorno. Las implicaciones prácticas estan en relación al conocimiento de las mejores opciones en el diseño para la automatización y acomodo de cultivos dentro de un invernadero.

\section{Metodología}

El proyecto se dividirá en etapas, para poder asegurar que se dará fin al proyecto, ya que se considera un proyecto a gran escala. Las etapas a considerar:

1. Diseño del sistema mecánico que permita al robot desplazarse por las mesas marcadas en su trayectoria.

2. Construcción y pruebas del mecanismo en prototipo a escala.

3. Desarrollo de la programación para trayectorias

4. Construcción del prototipo real de trabajo, utilizando celdas solares para asegurar su autonomía.

5. Diseño del sistema de control, tablillas y programación.

6. Estabilidad.

En la figura 1 se muestra el diseño de la distribución de las mesas y las plantas. El modelado de las estructuras se realizó utilizando el software SketchUp Pro-2018. Se tomó como base el plano descrito en la Figura 1.

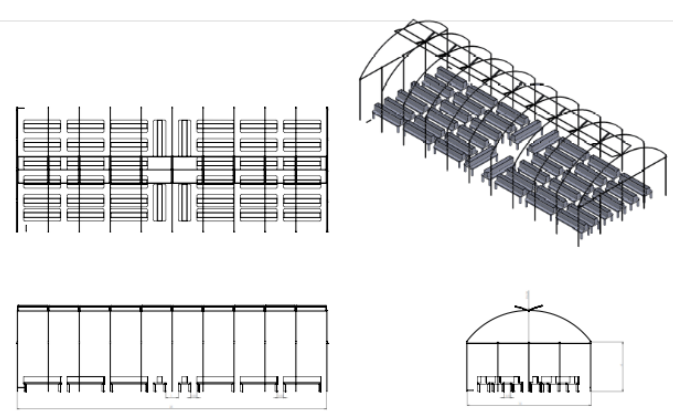

Figura 1 Distribución geométrica de mesas y plantas dentro del invernadero

Con base en esta distribución y con la consideración de instalación de sistemas de riego y las zonas de acceso y tráfico dentro del invernadero, se toma la determinación de seleccionar el carro robot que cumpla lo más posible con las trayectorias que se requiere para efectuar el proceso de fertilización. 
Como parte del desarrollo de las primeras etapas del proyecto se realiza el modelado de las estructuras del invernadero tipo, utilizando el software SketchUp Pro-2018. Se tomó como base el plano descrito en la Figura 1. En la figura 2 se muestra la arquitectura propuesta de un carro robot tipo Cruxent como apoyo en el diseño planteado para el robot fertirrigador, algunos de los módulos (electrónicos, eléctricos o mecánicos) son instalados directamente en el interior del chasis del robot, entregando así una arquitectura ideal de diseño, por su simplicidad, robustez y bajo costo.

La configuración básica del prototipo del robot se encuentra descrita a continuación: (1) torreta giratoria para conexión a tomas en mangueras. (2) conector de manguera. (3) sensores de presencia. (4) puerta de acceso a interior de cuerpo articulado delantero. (5) ruedas de tracción moldeadas en silicón duro blanco. (6) cuerpo articulado delantero. (7) motores de tracción y dirección delanteros. (8) cuerpo articulado posterior. (9) conector a bomba (10) base para dirigir manguera (11) cámara para reconocimiento de patrones (12) puerta de acceso a interior de cuerpo articulado posterior.

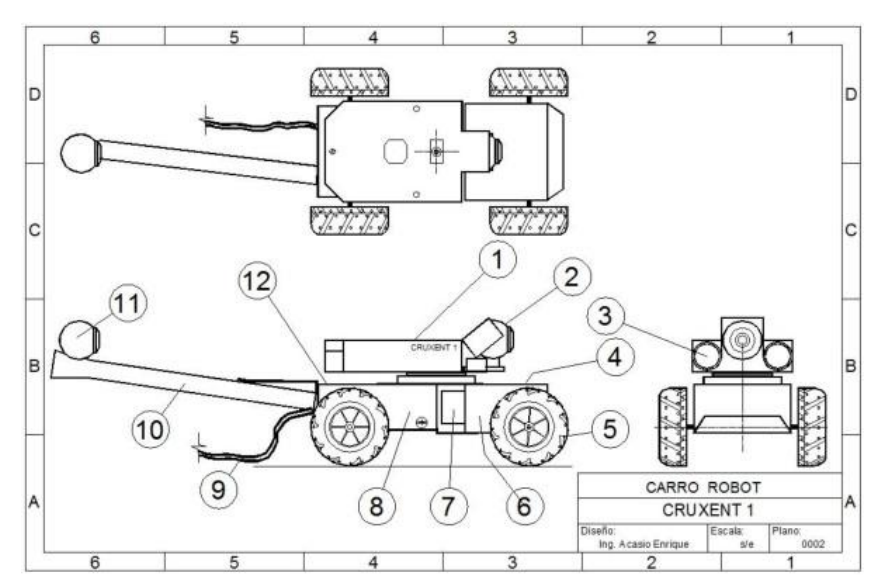

Figura 2 Robot fertirrigador

Asimismo se proponen las trayectoria de trabajo como se muestra en la figura 3 , en donde se genera una trayectoria perfectamente bien definida como se muestra en la figura $3 a$, mientras que en la $3 b$ la trayectoria en más compleja, esto se realiza con la finalidad de detectar que el móvil realmente responda al seguimiento de la línea, es importante mencionar que se trabajará con línea negra, y el lugar en donde se colocarán evitará un juego de luces que provoque que la trayectoria se pierda o que el robot confunda su dirección.

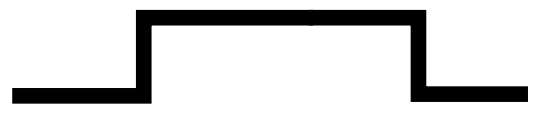

(a)

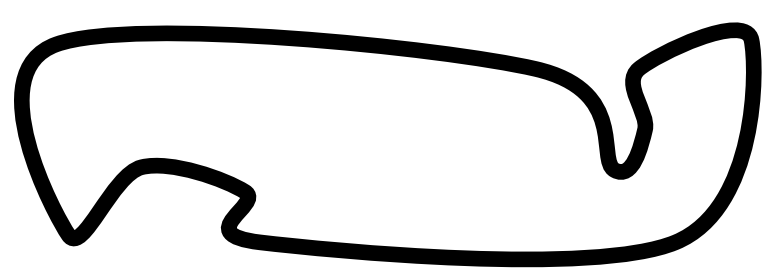

(b)

Figura 3 Trayectorias de prueba para el robot fertilizador

\section{Resultados}

Se modeló una estructura para invenadero, las dimensiones fueron modeladas arbitrariamente para esta fase inicial con base a los planos de la figura 1. Altura total: $7 \mathrm{~m}$. Se modelaron un total de 11 estructuras (Figura 3 y 4), únicamente las estructuras laterales tienen una serie de pilares (3 pilares intermedios). Las 9 estructuras estan corren a lo largo del invernadero modelado.

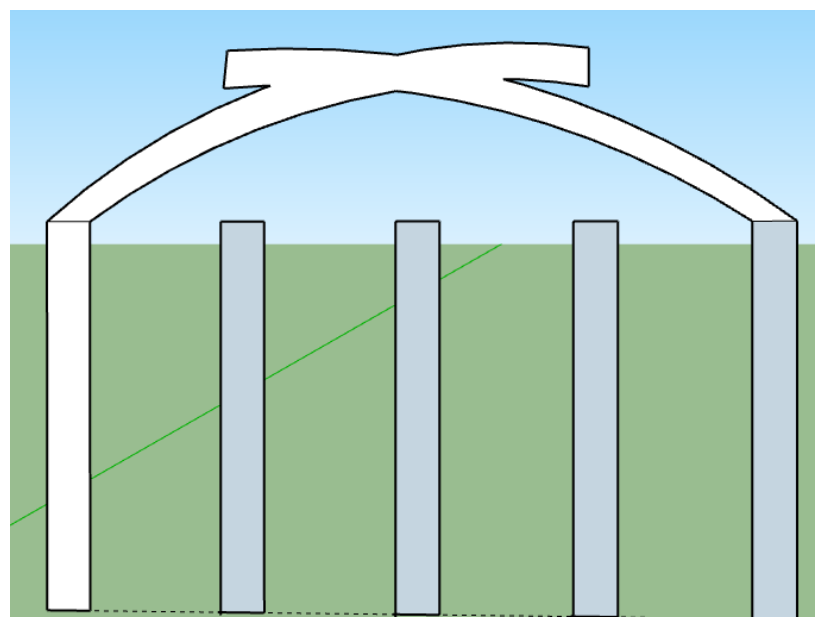

Figura 4 Características de los pilares de las estructuras laterales. Longitud total: 9 metros. Distancia entre pilares: 2.25 metros. Ancho del pilar: 10 centímetros. Altura del pilar: 4.90 metros

\section{Modelado para mesas de cultivo}

Las mesas fueron modeladas y distribuidas en 3 matrices, 2 de estas con un tamaño de $3 \times 3$ y 1 matriz con un tamaño de $2 \times 2$. Únicamente se fijo la altura de las mesas en el programa y diseño fijándola en 50 centimetros (Figura 6). 


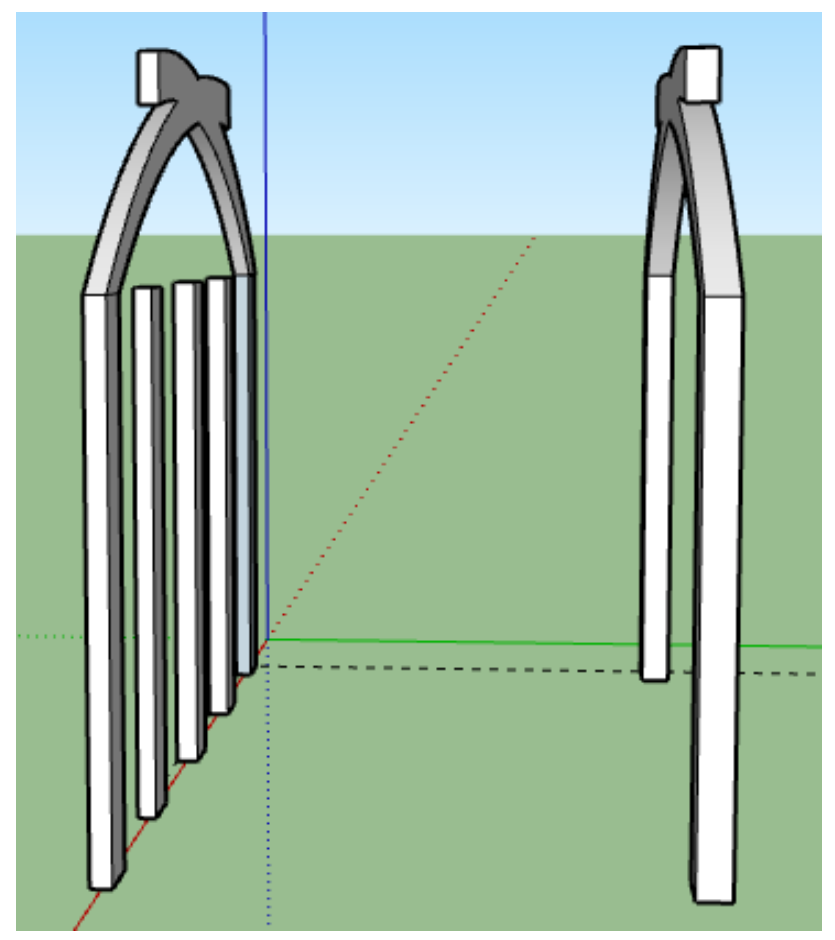

Figura 5 Distancia que separa las diferentes estructuras o es de 5 metros

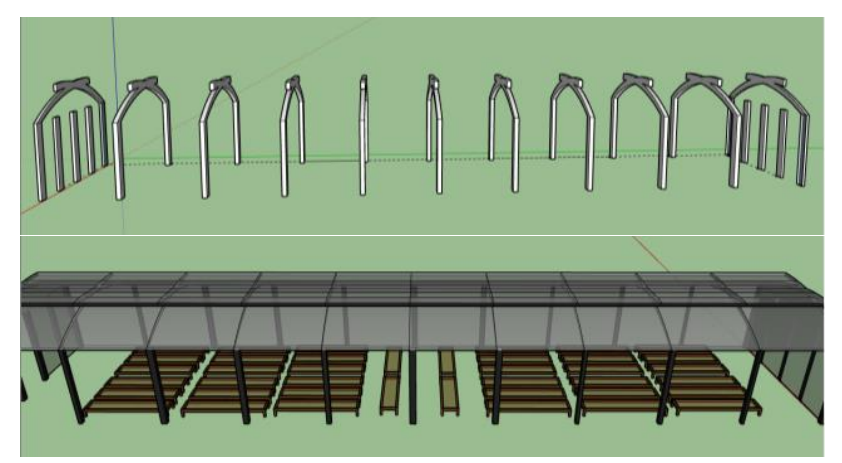

Figura 6 Andamiaje y estructuras totales para el modelo del Invernadero. Se modelo un total de 11 estructuras, fijando una longitud de 50 metros, para todo el andamiaje del invernadero

\section{Modelado Virtual del Robot}

Para la elaboración del robot, fueron utilizados 2 modelos proporcionados por la tienda en línea 3D Warehouse, estos modelos son gratuitos. En este caso, se utilizaron modelos para las llantas y cámaras. Una vez que se tienen esos elementos, se creó la estructura base para el robot, contando con unas medidas de:

\section{- $\quad$ Altura: $50 \mathrm{~cm}$. \\ - $\quad$ Longitud: 1.20 metros.}

La manera en que se realizarán los movimientos laterales será por medio de la siguiente estructura. Una de las cámaras será colocada en una estructura como la que se muestra en la siguiente figura 7.
Una segunda cámara será colocada en la parte trasera del robot, esta estructura tendrá unas medidas de $0.5 \mathrm{~m}$ y un ángulo $65^{\circ}$. Una vez terminada la estructura, se colocaron las llantas y las cámaras previamente descargadas, dando como resultado el siguiente modelo, contando con una altura total de 1.50 metros

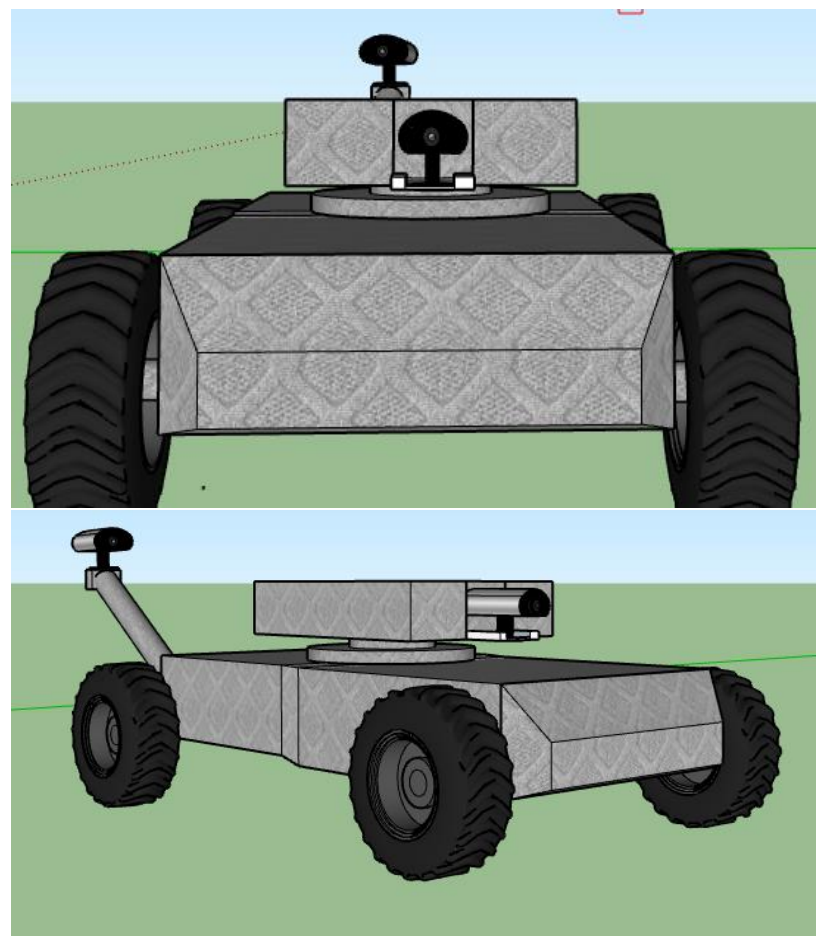

Figura 7 Modelo del robot virtual y ubicación de cámaras

Una vez terminados los modelos, deben de ser exportados como un modelo 3D para que puedan ser utilizados posteriormente en el software Unity, la manera de realizar esto se muestra a continuación. Para importar un elemento creado en SketchUp, basta con arrastrar el archivo y colocarlo en la parte inferior del software Unity, en el apartado que contiene el texto "Assets".

En este apartado se pueden colocar cualquier elemento que se quiera añadir al modelado (figura8), en este caso se agregaron unas pequeñas plantas para simular el cultivo del invernadero.

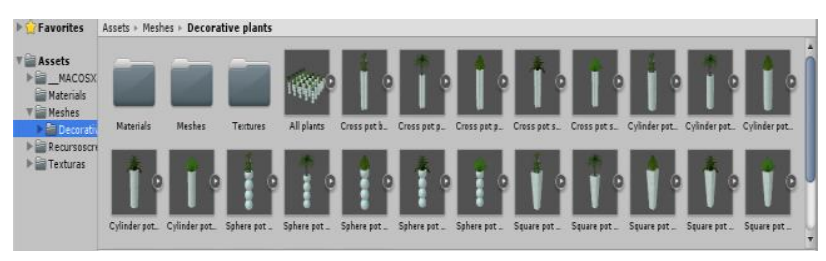

Figura 8 Modelado en software Unity del robot

BETANZOS-CASTILLO, Francisco, BECERRA-AMBRIZ, María Cecilia, MORÁN-HERNÁNDEZ, Juan y JIMÉNEZ-CAMPUZANO, Everardo. Algoritmo de seguimiento y planeación de trayectorias para un robot fertilizador en invernaderos. Revista de Simulación Computacional. 2019 
Para establecer el recorrido, se hizo uso del elemento "Game Object" y seleccionando los puntos por los cuales realizará su recorrido el robot El recorrido que realizará el robot, esta delimitado por las líneas amarillas que se pueden observar en las siguientes figuras.

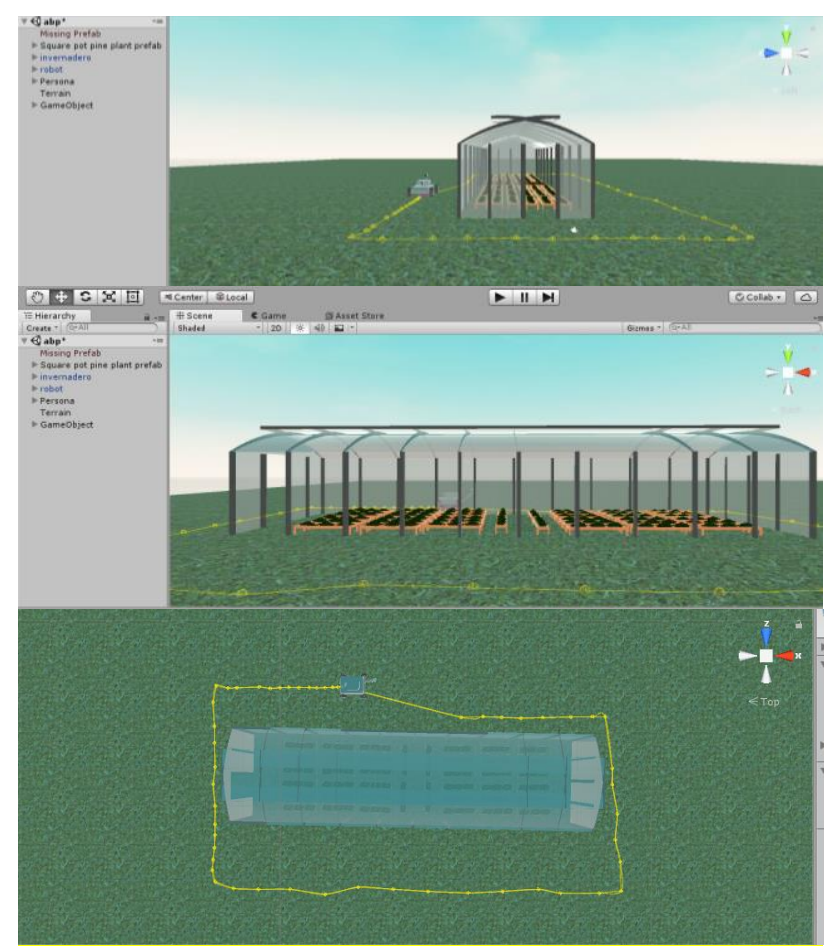

Figura 9 Trayectorias del robot virtual en Unity

\section{Conclusiones}

Con este desarrollo se puede concluir que el prototipo virtual construido cumple con las necesidades del seguimiento de las trayectorias, se probó en un ambiente similar al que se vivirá dentro del invernadero y se realizaron algunos ajustes en cuanto a la colocación de los sensores y cámaras.

Se pudo observar que resulta útil, el colocar sensores de proximidad, que asegura el que el robot no tenga choques con objetos que repentinamente puedan ubicarse a su paso, esto permite que el sistema no sufra descomposturas por impacto.

Se realizó la construcción del prototipo a tamaño real, y montar los sensores de proximidad y detección de línea negra. Se montó el sistema de conexión y desconexión a tubería. Este proceso se ha complicado un poco, ya que la falta de recursos necesarios lo ha vuelto muy lento, aun cuando el prototipo de prueba cumple con las maniobras de trayectorias y posición.
Se ha comprobado que la plataforma arduino es bastante robusta para el trabajo que va a desempeñar, lo que si se debe de cuidar es la parte de aislar el circuito para evitar que sufra de salpicaduras de agua y fertilizante, que remedie la formación de condensación, situación que ya se está resolviendo, ya se está construyendo las tablillas electrónicas que sustituirán a las protoboard para eficientar el espacio de trabajo.

La decisión de mecanizar el proceso de fertilización, permitirá mejorar la calidad del cultivo, de la misma forma permite disminuir la exposición de los trabajadores a sustancias que podrían resultar nocivos para su salud.

Se generó la simulación y se seleccionó el tipo de locomoción, una siguiente etapa será construir un prototipo que cumpla con las especificaciones señaladas, así mismo se le dotará de celdas solares para que presente una mayor autonomía, considerando que no siempre se cuenta con energía eléctrica en el lugar de ubicación del invernadero, además de que con este sistema se evitará el tender cables que puedan ocasionar un accidente.

\section{Referencias}

Bakker, T.; Asselt Vam, K.; Bontsema, J.; Müller, J.; Straten Van, G. (2010) Systematic Desing of an Autonomous Platform for Robotic Weeding. Jornal of Terramechanics, 47(2), pp.63-73.

Bell, T. (2000). Automatic Tractor Guidance Using Carrier-Phase Differential GPS. Computer and Eletronics in Agriculture. 25(1/2) , pp.53-66.

Bellis, M. (2009). A History of American Agriculture 1776-1990. Retrieved April 9, 2019, from

http://theinventors.org/library/inventors/blfarm1 .htm

Bonifaz, J., Happy, S., Linhares, B., \& Velasco, J. (2009). Mechatronics in the Evolution of Our Agriculture, 1-7.

Muhammad Faizan Siddiqui, Asim ur Rehman Khan, Neel Kanwal, Haider Mehdi, Aqib Noor, M. A. K. (2017). Automation and Monitoring of Greenhouse. IEEE Journal, (December), 1-14. 